\title{
Reproducibility of Trial-Based Functional MRI on Motor Imagery
}

\author{
S-S. Yoo ${ }^{1}$, H. M. O'Leary ${ }^{1}$, J-H. Lee ${ }^{1}$, N-K. Chen ${ }^{1}$, L. P. Panych ${ }^{1}$, H. Park ${ }^{2}$, F. A. Jolesz ${ }^{1}$ \\ ${ }^{1}$ Radiology, Brigham and Women's Hospital, Harvard Medical School, Boston, MA, United States, ${ }^{2}$ Electrical Engineering and Computer Science, KAIST, Daejon, \\ ChoongNam, Korea, Republic of
}

Introduction: The investigation of the reproducibility of functional MRI (fMRI) of human brain function is an important step in the quantification and analysis of paradigm-related brain activation. Most investigations examining the degree of reproducibility have been performed using various sensory motor and cognitive tasks that are overt/expressive [1]. Imagery and its fMRI detection/reproducibility have not been studied extensively. The potential application of imagery, for example, is to guide the optimal rehabilitation strategy for patients with brain damage (such as post-stroke recovery) by allowing patients to monitor and regulate their own brain function using fMRI neurofeedback [2]. We were motivated to evaluate the degree of reproducibility of the fMRI measurement during the motor imagery task using the trial-based design, which characterizes the neural activity over short time intervals (on the order of seconds).

Method: The study was conducted in accordance with the ethical guidelines. Ten (8 male) healthy volunteers between the ages of 19 and $49(27.9 \pm 8.9)$ were selected to participate in this study (9 right-handed). All experiments were performed using a 3 Tesla clinical MR scanner (GE Medical; TR/TE=1s/40ms, 64x64 matrix, $240 \mathrm{FOV}, 135-\mathrm{mm}$ axial slices). All subjects practiced the task 2-3 times prior to the scanning to reduce the variant activation patterns [1]. A total of $10 \mathrm{fMRI}$ scans (runs) were then administered repeatedly with about a 15-second gap between runs. During each run, there were two tasks that the subjects were asked to perform while in the magnet. The first task was a motor imagery task (MI) where the subjects were instructed to imagine squeezing their fist (dominant hand) 2 times per second. Each task lasted $60 \mathrm{~s}$ long with go occurring at $20 \mathrm{~s}$ and stop at $25 \mathrm{~s}$. The second task was a passive listening binaural, auditory task where the subjects were presented with sound for $60 \mathrm{~s}$. Amplitude-modulated (AM) auditory stimuli $(900 \mathrm{~Hz}$ tone, amplitude-modulated at $6 \mathrm{~Hz}$ with an amplitude range of $47.5 \mathrm{~dB} \pm 30 \% \mathrm{SPL}$ ) were presented during the control condition. During the stimulation trial, the auditory tones with both frequency- (modulated at $6 \mathrm{~Hz}$ with a frequency range of $900 \mathrm{~Hz} \pm 12 \%$ ) and amplitude-modulation (at $6 \mathrm{~Hz}$ with an amplitude range of $72.5 \mathrm{~dB} S P L \pm 30 \%$ ) were presented for $5 \mathrm{~s}$. The acquired data was reconstructed and analyzed using software developed in Matlab (Mathworks, MA). After the motion correction, a reference waveform which conforms to the given task paradigm was prepared using canonical hemodynamic response function obtained from SPM, and the pixel-by-pixel correlation coefficient was calculated with respect to the 60 temporal data points. Two regions-of-interest (ROls), the precentral gyrus (contralateral side of the hand clenching) for the motor imagery task and the bilateral auditory areas (Heschl's gyrus, area 41 and 42 in the Brodmann's area) were then delineated using the segmentation template from MRlcro (www.mricro.com ver.1.39). We measured two parameters, - (1) the size of the activation and (2) the magnitude of BOLD signal contrast - within the defined ROIs. The size of the activation was calculated by counting the number of activated voxels at $p$-values $<10^{-2}, 5 \times 10^{-3}, 10^{-3}, 5 \times 10^{-}$ ${ }^{4}$, and $10^{-4}$ within each ROI. In order to calculate the magnitude of the BOLD signal contrast, the signal time course from the voxels that survived the thresholding condition were first averaged. Then, the ratio between the amount of BOLD contrast measured during the task and the baseline signal level was calculated. These parameters were measured from 10 repeated runs for each volunteer. In order to examine the variability of the measured parameters across 10 runs for each subject, we calculated an index, 'intra-session variability' - the standard deviation of the mean activation parameters across 10 runs, subsequently normalized with respect to their mean (in percentage). This index acts as a measure of how much the size of activation and BOLD signal contrast deviate from each subject's norm. Therefore, a lower value of intra-session variability indicates better reproducibility.

Results: In order to examine the normal range of intra-session variations in terms of the size of activation and BOLD signal contrast across all 10 subjects and threshold conditions, the calculated intra-session variability indices at each threshold condition was averaged across the subjects and plotted in the Figure. The variability in terms of activation volume was greater in the motor imagery task (in white diamond) as compared to the auditory task (paired $t$-test, $\mathrm{p}<0.01$ ). However, this task-dependent difference was not apparent from the measurement of the BOLD signal contrast. We also examined the presence of the threshold dependence on the variability indicators using ANOVA, and found such dependencies were not evident in any of the tasks for either volume of activation or BOLD contrast (all exceeding $p>0.1$ ). The variation was greater in terms of the size of activation compared to the BOLD contrast in both motor imagery and auditory tasks. For example, most of the intra session-variability values exceeded $60 \%$ of the mean activation size; however variability of BOLD contrast was less than $40 \%$ for both task conditions across all subjects. Detailed statistical analysis showed that intrasession variability was lower in the BOLD signal contrast measurement as compared to the size of activation for both motor imagery (paired t-test; $\mathrm{p}<0.001, \quad d . f=9$ ) and auditory stimulation (paired $t$-test; $p<0.001, d . f=9$ ).
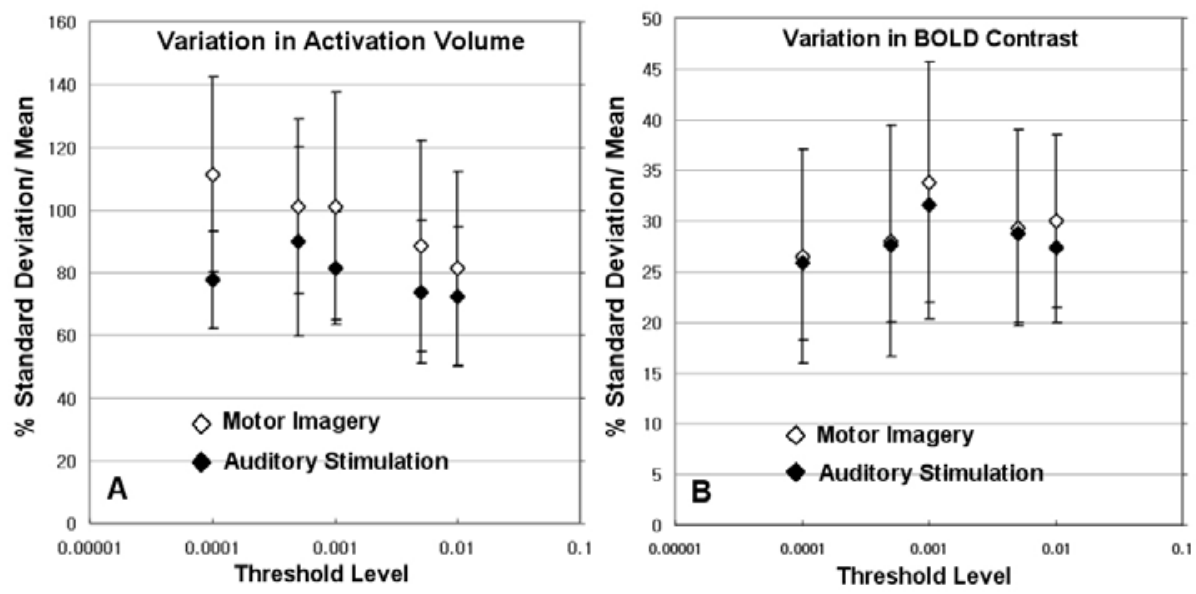

Fig. The group-averaged intra-session variability (as the stand deviation with respect to the mean inter session value out of 10 runs) for all 10 subjects in terms of the $(A)$ activation volume and (B) BOLD contrast, plotted for different threshold conditions (in log scale, x-axis).

Discussion: We found that the motor imagery and associated brain activation pattern, when administered in trial-based design, can be detected with reasonable reproducibility comparable to passive auditory stimulation/perception. Our data suggest that the BOLD signal contrast affords a much higher level of reproducibility over the volume of activation, therefore implying the utility of using the BOLD signal contrast as a measure of neurofeedback signal to the subject. Further investigation is necessary to examine the reproducibility of the repeated fMRI session data acquired on different days, i.e. inter-session reproducibility.

Reference: [1] McGonigle, et al(2000). Neuroimage, 11(6 Pt 1), 708-734.[2] deCharms et al. (2004) Neuroimage, 21(1), $436-443$. 